\title{
Notas sobre Thymelaea hirsuta (L.) Endl. y Thymelaea lythroides Barratte \& Murb. en la provincia de Sevilla (España)
}

\author{
José Manuel Herrera \& Íñigo Pulgar \\ Área de Botánica, Dpto. Biología Molecular e Ingeniería Bioquímica, Univ. Pablo de Olavide, Carretera de Utrera, Km 1. \\ 41013-Sevilla.
}

\section{Correspondencia}

Íñigo Pulgar.

E-mail: inigo.pulgar@gmail.com

Recibido: 2 junio 2020

Aceptado: 9 septiembre 2020

Publicado on-line: 6 octubre2020

Editado por: Andrés V. Pérez Latorre

\begin{abstract}
Resumen
Thymelaea lythroides es una especie amenazada de la que se conocen sólo dos poblaciones en la Península Ibérica. Se localiza una nueva población de la que se aportan datos sobre la vegetación circundante, la distribución espacial y la relación de sexos de los individuos que la conforman. También se aportan dos inventarios con Thymelaea hirsuta y se discute la serie de vegetación en la que aparece esta planta que resulta novedad para la provincia de Sevilla.
\end{abstract}

Palabras clave: especies amenazadas, corología, censos, vegetación.

\begin{abstract}
Notes on Thymelaea hirsuta (L.) Endl. and Thymelaea lythroides Barratte \& Murb. in Seville province (Spain).
\end{abstract}

\begin{abstract}
Thymelaea lythroides is a threatened species with only two known populations in the lberian Peninsula. A new population is located and data are provided on the surrounding vegetation, the spatial distribution and the sex ratio of the individuals that comprise it. Two releves are also provided with Thymelaea hirsuta and the vegetation series in which this plant appears, which is a novelty for the province of Seville, is discussed.
\end{abstract}

Key words: threatened species, chorology, censuses, vegetation.

\section{Thymelaea lythroides Barratte \& Murb.}

Hasta la fecha sólo se conocían dos localizaciones peninsulares de este iberonorteafricanismo. A la localidad clásica descubierta por Vicioso en la dehesa de Montepalacio (Paradas, Sevilla) (Pedrol, 1992, 1997; Romero-Zarco \& Albarreal, 1994; Roales, 1997), se sumó la distante del norte de Toledo (Vaquero de la Cruz, 2006). Encontramos una nueva población en la Campiña Baja de Sevilla en el término municipal de Marchena. Cuenta con dos subpoblaciones que distan entre sí $0,5 \mathrm{~km}$, que a su vez se encuentran separadas de la localidad clásica por una distancia de 7 y $8 \mathrm{~km}$ en línea recta respectivamente, lo que limita o impide el intercambio genético entre ellas (Iriondo et al. 2009). La primera subpoblación se encuentra muy fragmentada a lo largo de medio kilómetro de una vía pecuaria. En ella existen cuatro núcleos con 8, 5, 3 y 5 ejemplares, y 1 individuo aislado en posiciones intermedias. En total 22 individuos reproductores, 10 masculinos y 12 femeninos, habiéndose contabilizado ejemplares de ambos sexos en cada uno de los cuatro núcleos y siendo una planta femenina la que se encuentra aislada. Sólo se han observado dos ejemplares femeninos con alguna flor estaminada. No se han observado plántulas recientes del último año, aunque se han contabilizado cinco plantas jóvenes, de las cuales una no presenta flores. En dos de los núcleos $T$. Iythroides comparte el espacio con un reducido número de especies del matorral serial, un palmitar de Chamaerops humilis L. poco diverso que apenas incorpora esparragueras (Asparagus acutifolius L., Asparagus aphyllus L. y Asparagus albus L.) y con menor presencia Retama sphaerocarpa (L.) Boiss., Daphne gnidium L., Cistus salviifolius L. y Drimia maritima (L.) Stearn. En el resto de las localizaciones de esta población Thymelaea constituye el único manto leñoso sobre el terreno. La vegetación anual es principalmente nitrófila en toda la población. Lo mismo que en la localidad clásica, la vegetación potencial, es un alcornocal acidófilo sobre arenas (Romero-Zarco \& Albarreal, 1994) perteneciente al Myrto communis-Quercetum suberis Barbero, Quézel \& RivasMartínez. 1981 (Nieto Caldera et al. 1990; Pérez Latorre et al. 1994), aunque en este lugar esté prácticamente desaparecido y solo es posible visualizar algunos ejemplares de gran porte de Quercus suber L. y Quercus rotundifolia Lam. muy aislados entre sí. La segunda subpoblación también se localiza en una vía pecuaria y apenas cuenta con 4 individuos reproductores (2 machos y 2 hembras), constituyendo la única especie leñosa en un entorno donde predomina la vegetación anual sabulícola, siendo especialmente abundante Brassica oxyrrhina Coss. 
Ambas vías pecuarias presentan una anchura muy reducida en comparación con su anchura legal, debido principalmente a la ocupación de la misma por cultivos y edificaciones, por lo que ambas subpoblaciones están expuestas a un elevado riesgo de sufrir afecciones al encontrarse en suelo público de gran accesibilidad y alto grado de alteración y degradación. Además, es notable en algunos individuos el efecto del ramoneo por parte de roedores, principalmente por conejos.

Existen muchos argumentos científicos y legales que sugieren la conveniencia de conservar las nuevas poblaciones descubiertas. T. lythroides aparece en la lista roja de la flora vascular de España (Moreno, 2008) y en la de Andalucía (Cabezudo \& Talavera, 2005) con la categoría de CR B2ab(ii,iii). La Junta de Andalucía ha llevado a cabo actuaciones de conservación en la localidad clásica, siempre dentro de terrenos privados, que han consistido en la protección de la población mediante cerramiento o vallado de los individuos conocidos (Martín et al. 2013). En las nuevas poblaciones es de aplicación la Ley 3/1995, de 23 de marzo, de vías pecuarias, que entre sus fines (art. 3) obliga a conservar los elementos ambientales o culturalmente valiosos directamente vinculados a ellas, mediante la adopción de las medidas de protección y restauración necesarias (BOE-A-1995-7241). Fuera de los espacios más sólidamente protegidos (Parques Nacionales y Naturales, CEPA, LIFE, etc.) se han llevado a cabo distintas experiencias de protección de especies amenazadas (microreservas, custodia del territorio), que tienen en cuenta a la población local y que juzgamos interesantes para estas especies raras que sobreviven en entornos con un fuerte impacto agrario.

Sevilla, Marchena, Vereda de los Abrigosos. 30STG8823. 189 metros. Margen camino, sobre arenas. 15/01/2020. J.M. Herrera \& Í. Pulgar. UPOS 13550

Sevilla, Marchena, Cañada Real de Pruna/El Palmar. 30STG8825. 178 metros. Margen camino, sobre arenas. 15/03/2020. J.M. Herrera \& Í. Pulgar. UPOS 13551.

\section{Thymelaea hirsuta (L.) Endl.}

A la distribución peninsular aportada por Pedrol (1997), hay que sumar la cita de Sánchez-Gullón et al. (2011) en el litoral onubense. Según estos autores la distribución del taxón está ligada a los afloramientos de rocas calcáreas próximas a la línea de costa. Nuestra localidad en la Campiña Alta Sevillana se encuentra a una distancia de $76 \mathrm{~km}$ del mar y separado de él por las Subbéticas malacitanas y del sur de Sevilla. No conocemos otras citas sevillanas, ya que la de Albarreal \& Romero Zarco (1993) en Paradas es rectificada poco después (Romero Zarco \& Albarreal, 1994). La población de Osuna, con más de 100 individuos ocupa una ladera de orientación S-SE y zonas llanas adyacentes. Hemos levantado dos inventarios siguiendo la metodología fitosociológica de Braun Blanquet (1979) (Tabla 1) para recoger la diversidad vegetal de la comunidad con $T$. hirsuta; sólo se ha incorporado el índice de cobertura tal y como bien siendo habitual en los trabajos fitosociológicos publicados en los últimos años. El primer inventario refleja al matorral abierto con escasa presencia del taxón, mientras el segundo ha sido levantado en la zona de mayor densidad y con abundante retama. En ambos casos se trata de un matorral serial en el que asoman esporádicamente algunas encinas indicadoras de la vegetación potencial, un encinar basófilo subordinado a la asociación Paeonio coriaceae-Quercetum rotundifoliae Rivas-Martínez 1965 en su versión más termófila, subas. pistacietosum lentisci Pérez Latorre, Soriguer \& Cabezudo 2016 (Cabezudo et al. 2016), también tratada a nivel de Facies por Valle (2004) y Rivas Martínez (2011). El carácter serial de esta comunidad queda expresado florísticamente por la incorporación de taxones característicos de los tomillares de la asociación Teucrio IusitaniciCorydothymetum capitati, que colonizan suelos decapitados sobre sustratos básicos y quedan incorporados a la misma serie de vegetación en la Campiña Alta sevillana (Valle, 2004).

Sevilla, Osuna , El Calvario. 30SUG1923. 404 metros. Sobre arcillas, monte de caliza. 27/02/2020. J.M. Herrera \& Í. Pulgar. UPOS 13552.

Tabla 1. Inventarios con Thymelaea hirsuta Table 1. Inventories with Thymelaea hirsuta

\begin{tabular}{|c|c|c|}
\hline Inventario & 1 & 2 \\
\hline Superficie $\left(\mathrm{m}^{2}\right)$ & 50 & 80 \\
\hline Altitud (m) & 400 & 405 \\
\hline Cobertura (\%) & 70 & 60 \\
\hline Orientación & S & SE \\
\hline Pendiente (\%) & $<5$ & 20 \\
\hline № de especies & 12 & 15 \\
\hline Thymelaea hirsuta & 1 & 3 \\
\hline Cistus albidus & 3 & 1 \\
\hline Teucrium pseudochamaepitys & 2 & 2 \\
\hline Arisarum simorrhinum & 2 & 2 \\
\hline Thymbra capitata & 2 & 1 \\
\hline Ulex parviflorus subsp. parviflorus & 1 & 1 \\
\hline Rhamnus oleoides subsp. oleoides & 1 & 1 \\
\hline $\begin{array}{l}\text { Teucrium capitatum subsp. } \\
\text { capitatum }\end{array}$ & 1 & 1 \\
\hline Chamaerops humilis & 1 & 1 \\
\hline Phlomis purpurea & 1 & 1 \\
\hline Asparagus albus & 1 & . \\
\hline Quercus coccifera & 2 & . \\
\hline Daphne gnidium & $(r)$ & . \\
\hline Retama sphaerocarpa & . & 2 \\
\hline Olea europaea var. sylvestris & . & 1 \\
\hline Asparagus acutifolius & . & + \\
\hline Drimia maritima & & + \\
\hline Macrochloa tenacissima & . & $r$ \\
\hline
\end{tabular}

Localidades: 1 y 2. Sevilla, Osuna. El Calvario. 30SUG1918; 30SUG1919. 


\section{Agradecimientos}

A los revisores anónimos que con sus comentarios han contribuido a mejorar el escrito.

\section{Bibliografía}

Albareal, J. \& Romero Zarco, C. 1993. Novedades florísticas para la Campiña y Subbéticas sevillanas. Lagascalia 17(1): 190-193.

Braun Blanquet, J. 1979. Fitosociología. Ed. Blume. Madrid.

Cabezudo, B. \& Talavera, S. 2005 (coord.). Lista Roja de la flora vascular de Andalucía. Consejería de Medio Ambiente, Junta de Andalucía.

Cabezudo, B., Soriguer, C., García Sánchez, J. \& Pérez Latorre, V. 2016. Flora y vegetación de la Zona de Especial Conservación (ZEC) Sierra de Camarolos (Málaga, España). Acta Bot. Malacitana 41: 163-246.

Iriondo, J.M., Albert, M.J., Giménez Benavides, L., Domínguez Lozano, F. \& Escudero, A. (Eds.) 2009. Poblaciones en Peligro: Viabilidad demográfica de la Flora Vascular Amenazada de España. Dirección General de Medio Natural y Política Forestal. (Ministerio de Medio Ambiente, y Medio Rural y Marino), Madrid, 242 pp.

Martín, J.; Fernández, L. \& Urios, G. 2013. Los Bosques isla en Andalucía. Consejería de Medio Ambiente y Ordenación del Territorio, Junta de Andalucía, Sevilla, 192 pp.

Moreno, J.C., coord. 2008. Lista Roja 2008 de la flora vascular española. Dirección General de Medio Natural y Política Forestal (Ministerio de Medio Ambiente, y Medio Rural y Marino, y Sociedad Española de Biología de la Conservación de Plantas), Madrid, 86 pp.

Nieto Caldera, J.M., Pérez Latorre, A.V. \& Cabezudo, B. 1990. Datos sobre la vegetación silicícola de Andalucía. I. Acta Bot. Malacitana 15: 179-192.
Pedrol, J. 1992. Thymelaea lythroides Barratte \& Murb. (Thymelaeaceae), ¿planta europea? In Notulae taxinomicae, chorologicae, nomenclaturales, bibliographicae aut philologicae in opus "Flora Iberica" intendentes. Anales Jard. Bot. Madrid 50(2): 278.

Pedrol, J. 1997. Thymelaea Mill. En Nieto (ed.), Flora Iberica 8: 42-69. Real Jardín Botánico-CSIC. Madrid.

Pérez Latorre, A.V., Nieto Caldera, J.M. \& Cabezudo, B. 1994. Datos sobre la vegetación de Andalucía. III. Series de vegetación caracterizadas por Quercus suber L. Acta Bot. Malacitana 19: 169183.

Rivas Martínez, S. 2011. Mapa de series, geoseries y geopermaseries de vegetación de España. Itinera Geobot. 18(1): 5-424.

Roales, J. 1997. Contribución al conocimiento de la flora de Sevilla: novedades corológicas para la provincia. Lagascalia 20(1): 129-149.

Romero Zarco, C. \& Albarreal, J. 1994. Thymelaea lythroides Barratte \& Murb. (Thymelaeaceae), planta europea. En notulae taxinomicae, chorologicae, nomenclaturales, bibliographicae aut philologicae in opus "Flora Iberica" intendentes. Anales Jard. Bot. Madrid 52(1): 125.

Sánchez Gullón, E., Rodríguez Rubio, P., De Las Heras, M.A. \& Muñoz, A. 2011. Novedades florísticas para el litoral de Huelva y provincia. IV. Lagascalia 31: 216-220.

Valle, F. (coord.). 2004. Datos botánicos aplicados a la Gestión del Medio Natural Andaluz, II: Series de Vegetación. Consejería de Medio Ambiente. Junta de Andalucía.

Vaquero de la Cruz, J. 2006. Plantas vasculares protegidas de la "Reserva Fluvial de los Sotos del río Guadyerbas y arenales del Baldío de Velada" (Toledo, España). Bol. R. Soc. Esp. Hist. Nat. (Sec. Biol.) 101 (1-4): 13-18. 